\title{
Improving the effectivity of residual based a posteriori error estimates using a statistical approach
}

\author{
V.J. Ervin *, L.N. Ntasin \\ Department of Mathematical Sciences, Clemson University, Clemson, SC 29634-0975, USA
}

Received 5 April 2004; received in revised form 16 November 2004; accepted 15 February 2005

8 Abstract

9 For the approximation of differential equations residual based error estimates provide upper bounds (usually gross 10 over estimates) to the true error. In this paper we present a procedure for determining values for the constants in the a 11 posteriori estimates which yield accurate estimates to the true error. Numerical experiments demonstrating the effective12 ness of the method are given.

13 (c) 2005 Elsevier B.V. All rights reserved.

14 AMS classification: $65 \mathrm{~N} 30$

15 Keywords: A posteriori; Error; Effectivity index; Non-linear least squares

\section{1. Introduction}

A posteriori error estimation is concerned with determining the accuracy of a computed approximation $\tilde{u}$. The obvious difficulty is that the true solution $u$ is unknown. In the approximation of a linear system, $A \underline{x}=b$, an indication of how "close" an approximation $\underline{\tilde{x}}$ is to $\underline{x}$ can be found by computing the size of

21 the residual vector $\underline{r}$, where $\underline{r}=\underline{b}-A \underline{\tilde{x}}$. This approach when applied in the numerical approximation of differential equations leads to residual based a posteriori error estimates (see [9]). These estimates are constructed as upper bounds and, when possible, lower bounds or local lower bounds. These bounds contain multiplicative constants that depend on the true solution, the domain, and the interpolation properties of the approximating spaces. Computing the exact values for these constants is equivalent to comput-

\footnotetext{
* Corresponding author.

E-mail address: vjervin@clemson.edu (V.J. Ervin).
} 
ing the true solution to the differential equation, which is virtually impossible in most, if not all, cases. Arbitrarily assigning values to these constants turns the a posteriori error estimates into error indicators. Such indicators are useful in providing comparative information about the error but do not provide accurate quantitative estimates for the true error. In this paper we present a method for improving the effectiveness of these error indicators. Using statistical techniques the method applies a non-linear least squares approach to determine model parameters, using as data a sequence of approximate solutions. Using the values of these parameters the true error in the approximate solution can then be estimated.

This paper is organized as follows. Section 2 begins by describing the general setting for the differential equation and the a posteriori error estimate. In Section 2.1 the construction of the sequence of approximate solutions is described. The procedure for determining the model parameters is presented in Section 2.2. Section 3 contains examples which demonstrate the effectiveness of the method for both linear and non-linear problems.

\section{Problem definition}

Consider a general differential operator $\mathscr{L}$ that defines a system of differential equations of the form

$$
\mathscr{L} u=f \quad \text { in } \Omega, \quad \text { and } u=g \text { on } \Gamma,
$$

where $\Omega \subset \mathbb{R}^{n},(n=2,3)$ is a polygonal domain with boundary $\Gamma$. Let $u_{h} \in \mathscr{X}_{h}$ be a finite element (FE) approximation to the solution $u \in \mathscr{X}$ of (2.1), where $\mathscr{X}_{h}$ is a finite dimensional subspace of $\mathscr{X}$ with spatial mesh parameter $h$. Assume that (2.1) fits the general framework presented by Verfürth in [9] for constructing residual based a posteriori error estimates. Using this framework a residual based a posteriori error estimate for (2.1) can be constructed in the general form

$$
\left\|u-u_{h}\right\|_{\mathscr{X}} \leqslant c_{1} \mathbf{R}_{1}+c_{2} \mathbf{R}_{2}+c_{3} \mathbf{R}_{3}+c_{4} \mathbf{R}_{4}
$$

where $c_{1}, c_{2}, c_{3}$ and $c_{4}$ are constants, and $\mathbf{R}_{1}, \mathbf{R}_{2}, \mathbf{R}_{3}$ and $\mathbf{R}_{4}$ represent the "strong form" residual, the consistency error, the oscillation error, and the residual of the approximating algebraic system, respectively. The "strong form" residual represents the residual of the governing equation (defined by $\left(\mathscr{L} u_{h}-f\right)$ ) plus the edge jump terms which result from rewriting the weak form as a strong form. The consistency error correspond to the regularization procedure used in computing an approximate solution $u_{h}$. The oscillation error comes from approximating the forcing term on the right hand side of (2.1) in a finite dimensional space (for example piecewise linears). The residual of the algebraic system measures the error in the solution of the algebraic system of approximating equations.

The oscillation error, $\mathbf{R}_{3}$, is usually a higher order term when compared to the "strong form" residual and the consistency error. If the approximating algebraic system is solved up to round off error then $\mathbf{R}_{4}$ is also negligible, relative to the "strong form" residual and the consistency error terms. So, under appropriate conditions $\mathbf{R}_{3}$ and $\mathbf{R}_{4}$ both have little influence on the a posteriori error estimate (2.2).

For low order methods, Carstensen and Verfürth [2] showed that edge jump terms dominate the a posteriori error estimates for elliptic problems. Also, for a wide variety of stabilization techniques $\mathbf{R}_{1}$ bounds $\mathbf{R}_{2}$ from above (see for example [4]). Thus, in those cases where $\mathbf{R}_{1}$ either dominates, or bounds $\mathbf{R}_{2}$, the a posteriori error estimate reduces to

$$
\left\|u-u_{h}\right\|_{\mathscr{X}} \leqslant c_{1} \mathbf{R}_{1}=c_{1}\left\{\sum_{T} \eta_{T}\left(u_{h}\right)^{2}\right\}^{1 / 2} .
$$


70 In (2.3) $\eta_{T}$ represents the local "strong form" residual of (2.1) plus the jump term of $u_{h}$ along the boundaries of the mesh element $T$ with its neighbors.

In general, if the mathematically computable values for the constants $c_{1}, c_{2}, c_{3}, c_{4}$ were used in (2.2) and (2.3), a gross over-estimate for the true error in the approximate solution would result. This is because (2.2) must account for the worst case scenerio at each step of the derivation. Ideally, we want a value for $c_{1}$ in (2.3) that satisfies the equality part of the inequality, i.e. a value of $c_{1}$, say $c^{*}$, such that

$$
\left\|u-u_{h}\right\|_{\mathscr{X}} \approx c^{*} \mathbf{R}_{1}=c^{*}\left\{\sum_{T} \eta_{T}\left(u_{h}\right)^{2}\right\}^{1 / 2} .
$$

79 For some problems, e.g. the Poisson problem, it can be shown that $c_{1}$ depends on the minimum angle of the

\section{Remarks.} mesh, the coercivity and continuity constants for the problem, and the interpolation properties of the approximating spaces (see [2]). For non-linear problems, $c_{1}$ also depends on the norm of the inverse of the linearized operator about the true solution (see [9]).

In general, the one parameter model (2.4) was not sufficient to accurately estimate the true error in the approximation (see Example 4, Fig. 6). We therefore consider a two parameter model described in the following assumption.

Assumption A. Given a general problem of the form (2.1) for which the error estimate (2.3) is valid, then there exists positive constants $c^{*}$ and $\theta$ such that

$$
\left\|u-u_{h}\right\|_{\mathscr{X}}=c^{*} \mathbf{R}_{1}^{\theta}=c^{*}\left\{\sum_{T} \eta_{T}\left(u_{h}\right)^{2}\right\}^{\theta / 2} .
$$

The objective of this paper is to develop a method for estimating $c^{*}$ and $\theta$ from data generated through a sequence of approximate solutions.

1. We have investigated including $\mathbf{R}_{2}$ and/or $\mathbf{R}_{3}$ in (2.5). Data exploration and preliminary statistical analysis showed strong collinearity between all three variables $\left(\mathbf{R}_{1}, \mathbf{R}_{2}, \mathbf{R}_{3}\right)$. With high levels of collinearity, a multiple regression model loses its ability to show the relative importance of the effects of different predictor variables on the response variable. Thus, small changes in the data may cause large fluctuations in the predicted variable; an undesirable effect. A possible remedy for this effect is to drop variables associated with less significant regression coefficients from the model. In our investigation $\mathbf{R}_{1}$ was consistently associated with the most significant regression coefficient.

2. The asymptotic value for $\theta$ in (2.5) is 1 , which comes from the upper bound estimate (2.3). However, our interest in this paper is on accurately estimating the error, not giving an upper bound for the error, in a practical computation. For such computations the asymptotic values for the constants in the upper bound are generally not the best choice to accurately estimate the error. (Clearly, for computations on a sufficiently fine mesh the value for $\theta$ must be approximately 1.)

3. A completely rigorous mathematical analysis for an equality estimate for the error is not possible. (For a rigorous mathematical analysis of an upper bound for the error see, for example [2,4,9].) Following in Sections 2.1 and 2.2 we present the mathematical motivation for the proposed procedure for constructing a posteriori error estimates. 


\section{2.1. Construction of a sequence of approximate solutions}

112 For a given problem, for which the a posteriori error estimate (2.3) holds, let $\mathscr{X}_{h, i} \subset \mathscr{X}$ and $\Pi_{h, i}=\Pi_{h, i}(\Omega)$, $113 i=1, \ldots, n+1$, represent a sequence of successively generated finite dimensional spaces and meshes (uni114 form or adaptive refinements), respectively. Let $u_{1}, u_{2}, \ldots, u_{n+1}$ represent the corresponding sequence of 115 approximate solutions to (2.1) computed using the meshes $\Pi_{h, i}, i=1, \ldots, n+1$. Also, for ease of notation, 116 let

$$
\eta\left(u_{i}, \Pi_{h, i}\right):=\left\{\sum_{T \in \Pi_{h, i}} \eta_{T}\left(u_{h}\right)^{2}\right\}^{1 / 2}
$$

119 and let

$$
\operatorname{osc}\left(f, \Pi_{h, i}\right):=c_{3} \mathbf{R}_{3}+c_{4} \mathbf{R}_{4},
$$

122 where $\mathbf{R}_{3}$ and $\mathbf{R}_{4}$ represent the oscillation error and the error in solving the algebraic system corresponding 123 to the mesh $\Pi_{h, i}$, respectively.

124 We make the following two assumptions [7].

125 Assumption I (Marking strategy). For a given mesh $\Pi_{h, i}, i=1, \ldots, n+1$, there exists a submesh, $126 \widehat{\Pi}_{h, i} \subset \Pi_{h, i}$ such that

127 1. $\eta\left(u_{i}, \widehat{\Pi}_{h, i}\right) \geqslant \mu \eta\left(u_{i}, \Pi_{h, i}\right)$,

128 2. $\operatorname{osc}\left(f, \widehat{\Pi}_{h, i}\right) \geqslant \operatorname{vosc}\left(f, \Pi_{h, i}\right)$,

129 where $0<\mu, v<1$.

130 Assumption II (Refinement strategy). The refinement strategy used to generate successive approximations 131 satisfies:

132 1. $\mathscr{X}_{h, i} \subset \mathscr{X}_{h, i+1}$ for $i=1, \ldots, n$,

133 2. If $T$ is an element of $\Pi_{h, i}$ marked for refinement by the marking strategy, then when $T$ is refined, the 134 refinement process

138 (a) generates at least one interior node in $T$,

(b) generates at least one interior node on each of the faces of $T$.

139 In [7] it was demonstrated that Assumptions I and II are necessary (may not be sufficient) to guarantee 140 an asymptotically convergent sequence of discrete approximations.

141 Assume that the sequence of approximate solutions $u_{1}, u_{2}, \ldots, u_{n+1}$ is asymptotically convergent (with re-

142 spect to the norm $\left.\|\cdot\|_{x}\right)$ to $u$. Next, define the sequence, $\left\{Y_{i}\right\}, i=1, \ldots, n$, by

$$
\begin{aligned}
Y_{1}:= & \left\|u_{2}-u_{1}\right\|_{\mathscr{X}}, \\
Y_{2}:= & \left\|u_{3}-u_{2}\right\|_{\mathscr{X}}, \\
& \cdots \cdots \\
Y_{n}:= & \left\|u_{n+1}-u_{n}\right\|_{\mathscr{X}} .
\end{aligned}
$$

146 In addition, let $\left\{Z_{i}\right\}, i=1, \ldots, n$, be given by 


$$
\begin{aligned}
Z_{1}:= & \left|\left\|u-u_{1}\right\|_{\mathscr{X}}^{2}-\left\|u-u_{2}\right\|_{\mathscr{X}}^{2}\right|^{1 / 2}, \\
Z_{2}:= & \left|\left\|u-u_{2}\right\|_{\mathscr{X}}^{2}-\left\|u-u_{3}\right\|_{\mathscr{X}}^{2}\right|^{1 / 2}, \\
& \cdots \cdots \\
Z_{n}:= & \left|\left\|u-u_{n}\right\|_{\mathscr{X}}^{2}-\left\|u-u_{n+1}\right\|_{\mathscr{X}}^{2}\right|^{1 / 2} .
\end{aligned}
$$

149 Assumptions I and II guarantee that progress is being made at each step, i.e. $u_{i+1}$ is a better approximation

150 to $u$ than $u_{i}$. Additionally these assumptions guarantee that stagnation does not occur in the approximation

151 process. That is, for $i=1, \ldots, n$ there is no $i$ such that

152

1. $\left\|u_{i+1}-u_{i}\right\|_{\mathscr{X}}=0$,

2. $\left|\left\|u-u_{i}\right\|_{\mathscr{X}}^{2}-\left\|u-u_{i+1}\right\|_{\mathscr{X}}^{2}\right|^{1 / 2}=0$.

In this setting the sequences $\left\{Z_{i}\right\}$ and $\left\{Y_{i}\right\}$ are guaranteed to converge to zero.

157 Next, let $\mathscr{X}_{h, i}$ and $\mathscr{X}_{h, i+1}$ represent two finite element spaces such that $\mathscr{X}_{h, i} \subset \mathscr{X}_{h, i+1} \subset \mathscr{X}$ and assume that $\|\cdot\|_{\mathscr{X}}$ is an inner product norm. Define $\bar{u}_{i}$ and $\bar{u}_{i+1}$ as the orthogonal projections of $u$ with respect to the norm $\|\cdot\|_{\mathscr{X}}$ in the spaces $\mathscr{X}_{h, i}$ and $\mathscr{X}_{h, i+1}$, respectively. Also, let $a=u-\bar{u}_{i}$ and $b=u-\bar{u}_{i+1}$ then

$$
\begin{aligned}
& (a-b, a-b)_{\mathscr{X}}=(a, a)_{\mathscr{X}}+(b, b)_{\mathscr{X}}-2(a, b)_{\mathscr{X}}, \\
& \|a-b\|_{\mathscr{X}}^{2}=\|a\|_{\mathscr{X}}^{2}+\|b\|_{\mathscr{X}}^{2}-2(a, b)_{\mathscr{X}} .
\end{aligned}
$$

162 Note that $(a-b)$ is orthogonal to $b$. Consequently,

$$
\|a-b\|_{\mathscr{X}}^{2}=\|a\|_{\mathscr{X}}^{2}+\|b\|_{\mathscr{X}}^{2}-2(a-b, b)_{\mathscr{X}}-2(b, b)_{\mathscr{X}}=\|a\|_{\mathscr{X}}^{2}-\|b\|_{\mathscr{X}}^{2} .
$$

165 If we let $u_{i}$ and $u_{i+1}$ be finite element approximations of $u$ in $\mathscr{X}_{h, i}$ and $\mathscr{X}_{h, i+1}$, respectively and assume that $166 u_{i} \approx \bar{u}_{i}$ and $u_{i+1} \approx \bar{u}_{i+1}$ then, for $a \approx u-u_{i}$ and $b \approx u-u_{i+1}$

$$
\|a-b\|_{\mathscr{X}}^{2} \approx\|a\|_{\mathscr{X}}^{2}-\|b\|_{\mathscr{X}}^{2} \text {. }
$$

170 Using (2.5) and (2.10), we have for $1 \leqslant i \leqslant n$

$$
\begin{aligned}
\left\|u_{i}-u_{i+1}\right\|_{\mathscr{X}}^{2} & \approx\left\|u-u_{i}\right\|_{\mathscr{X}}^{2}-\left\|u-u_{i+1}\right\|_{\mathscr{X}}^{2} \\
& =c^{* 2}\left\{\eta\left(u_{i}, \Pi_{h, i}\right)^{2 \theta}-\eta\left(u_{i+1}, \Pi_{h, i+1}\right)^{2 \theta}\right\}, \\
\left\|u_{i}-u_{i+1}\right\|_{\mathscr{X}} & \approx c^{*}\left|\eta\left(u_{i}, \Pi_{h, i}\right)^{2 \theta}-\eta\left(u_{i+1}, \Pi_{h, i+1}\right)^{2 \theta}\right|^{1 / 2} .
\end{aligned}
$$

174 In compact form we can then write the resulting system of equations as

$$
\left\{Y_{i}\right\} \approx c^{*}\left\{X_{i}(\theta)\right\}
$$

178 where

$$
X_{i}(\theta):=\left|\eta\left(u_{i}, \Pi_{h, i}\right)^{2 \theta}-\eta\left(u_{i+1}, \Pi_{h, i+1}\right)^{2 \theta}\right|^{1 / 2}=\left|\mathbf{R}_{1_{i}}^{2 \theta}-\mathbf{R}_{1_{i+1}}^{2 \theta}\right|^{1 / 2} .
$$

182 Note that for any given problem, $\left\{Y_{i}\right\}$ is computable from the sequence of approximate solutions while $183\left\{X_{i}\right\}$ is computable up to $\theta$ from the sequence of approximate solutions and the given problem data. 184 The parameters $c^{*}$ and $\theta$ in (2.12) are the same as the constants in (2.5). Thus, approximating values for $185 c^{*}$ and $\theta$ that yield an equality or almost an equality in (2.12) will give us approximate values for $c^{*}$ and $186 \theta$ that are usable in (2.5). Our main objective is therefore to develop a technique for determining values 
187 for $c^{*}$ and $\theta$ such that an approximate equality holds in (2.12) for a given problem and a sequence of approximate solutions.

In the case where (2.1) describes a linear second order elliptic operator which has an underlying inner 190 product, we have the following lemma.

191 Lemma 2.1 [7]. Suppose that the norm $\|\cdot\|_{\mathscr{X}}$ is defined by $\|v\|_{\mathscr{X}}^{2}:=a(v, v)$, where $a(\cdot, \cdot)$ represents the bilinear form corresponding to the left hand side of (2.1). If $\Pi_{h}$ is a refinement of $\Pi_{H}$ such that $\mathscr{X}_{H} \subset \mathscr{X}_{h} \subset \mathscr{X}_{\text {, }}$ where $\mathscr{X}_{H}$ and $\mathscr{X}_{h}$ are the finite element spaces corresponding to $\Pi_{H}$ and $\Pi_{h}$ respectively, then the following relation holds:

$$
\left\|u_{H}-u_{h}\right\|_{\mathscr{X}}^{2}=\left\|u-u_{H}\right\|_{\mathscr{X}}^{2}-\left\|u-u_{h}\right\|_{\mathscr{X}}^{2} .
$$

217 by the functional

$$
\mathscr{L}\left(c^{*}, \theta, \sigma^{2}\right):=\frac{1}{\left(2 \pi \sigma^{2}\right)^{n / 2}} \exp \left[-\frac{1}{2 \sigma^{2}} \sum_{i=1}^{n}\left[Y_{i}-c^{*} X_{i}(\theta)\right]^{2}\right],
$$

220 where $\sigma^{2}$ is the variance of $\varepsilon_{i}$ 's. To obtain the least squares line of best-fit of the form (2.15) for a given data 221 set, it is straight forward to observe that the least squares line is the solution to the problem

$$
\max _{c^{*}>0, \theta>0}\left\{\mathscr{L}\left(c^{*}, \theta, \sigma^{2}\right)\right\}=\max _{c^{*}>0, \theta>0}\left\{\frac{1}{\left(2 \pi \sigma^{2}\right)^{n / 2}} \exp \left[-\frac{1}{2 \sigma^{2}} \sum_{i=1}^{n}\left[Y_{i}-c^{*} X_{i}(\theta)\right]^{2}\right]\right\} .
$$

225 The procedure for finding $c^{*}$ and $\theta$ that maximizes $\mathscr{L}\left(c^{*}, \theta, \sigma^{2}\right)$ is known as the method of maximum like226 lihood. A priori knowledge of our data source suggest that $c^{*}>0$ and $\theta>0$. Thus, for any given data set, 227 appropriate parameter estimates $\widehat{c^{*}}$ and $\widehat{\theta}$ should be positive.

228 An underlying assumption of the model is that the $\varepsilon_{i}$ 's are independent and normally distributed with 229 mean zero and constant variance $\sigma^{2}$. Having determined the parameter estimates $\widehat{c^{*}}$ and $\widehat{\theta}$, this assumption 230 can be investigated by plotting the model residuals, $r_{i}=Y_{i}-\widehat{c^{*}} X_{i}(\widehat{\theta})$ against the $Y$-values (See Fig. 1). 

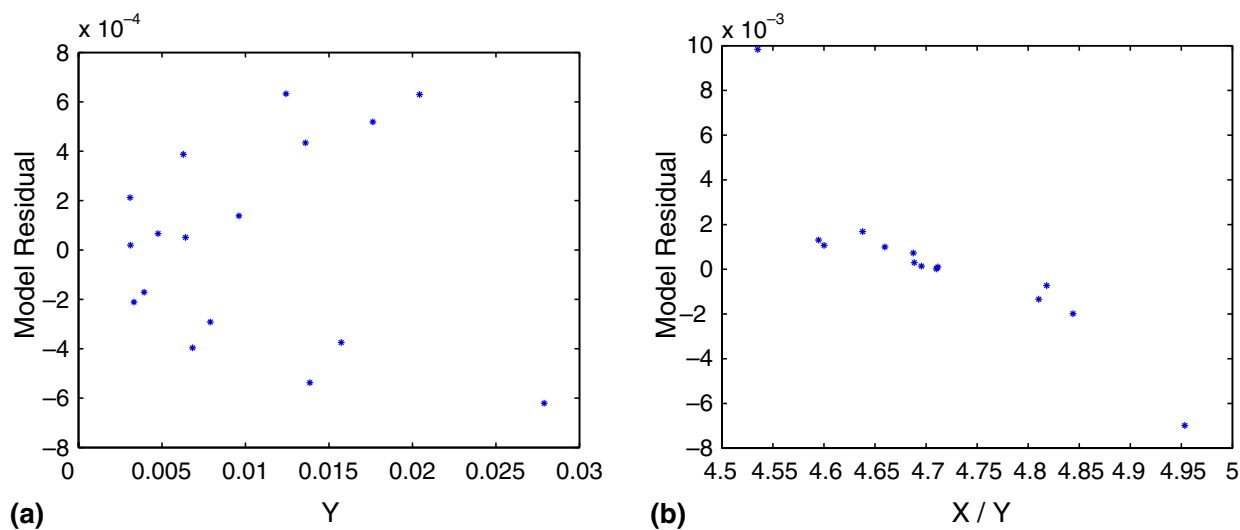

Fig. 1. Sample residue plots: (a) Sample residual plot for (2.15) and (b) sample residual plot for (2.20).

231 These plots, in general, have a "megaphone" shape indicating that the error variance $\sigma^{2}$ is not constant but grows with increasing $Y$-values. This violates the underlying assumption of constant variance.

Before applying a transformation to the likelihood estimator, with the objective of satisfying the normality requirement, let us examine the underlying objectives.

Consider the two models below:

$$
\begin{array}{ll}
\text { Model A : } & e=c^{*} \mathbf{R}_{1}^{\theta}+\varepsilon_{E}, \\
\text { Model } \mathbf{B}: & Y=c^{*} X(\theta)+\varepsilon_{Y},
\end{array}
$$

where $e=\left\|u-u_{h}\right\|_{\mathscr{X}}$ is the true error, $\varepsilon_{E}$ and $\varepsilon_{Y}$ represent the Models $\mathbf{A}$ and $\mathbf{B}$ residuals, respectively. Given appropriate values for $c^{*}$ and $\theta$ we use Model $\mathbf{A}$ to estimate the true error in a given approximate solution $u_{h}$. Since the $e_{i}$ 's are not known we cannot estimate $c^{*}$ and $\theta$ through Model A. So, we use Model B to estimate appropriate values for $c^{*}$ and $\theta$ that can be used in Model A. Satisfying the normality assumption in Model $\mathbf{B}$ may not be sufficient for obtaining values of $c^{*}$ and $\theta$ that are optimal for Model A.

First, let us address the "megaphone" shaped residual plots of Model B. Due to the fact that the data $\left(\left\{X_{i}\right\}\right.$ and $\left.\left\{Y_{i}\right\}\right)$ usually spans several orders of magnitude, absolute errors from Model $\mathbf{B}$ will most likely show the "megaphone" shaped residual plots. Through numerical experiments, the relationship between $\left|\varepsilon_{Y}\right|$ and the predicted $Y$-values, $\widehat{Y}$, is in general linear. This has led to the transformation of Model $\mathbf{B}$ as

$$
\bar{Y}=c^{*} \bar{X}(\theta)+\bar{\varepsilon}_{Y},
$$

where $\bar{Y}_{i}=1$, and $\bar{X}_{i}(\theta)=X_{i}(\theta) / Y_{i}$.

The corresponding residuals for (2.20) are plotted in Fig. 1(b). With the exception of the two largest (in magnitude) values, the residues lie in a band centered about zero, indicating the assumption of $\bar{\varepsilon}_{Y}$ having constant variance is reasonable. Displayed in Fig. 2 is a normal probability plot for the residues of (2.20). Fig. 2(a) is a normal probability plot using all the residual values; Fig. 2(b) a normal probability plot with the two largest (in magnitude) residues omitted. In a normal probability plot the nearer the points are to lying on a straight line the more likely the underlying distribution is close to a normal probability distribution. Based on Fig. 2(b), the assumption that $\bar{\varepsilon}_{Y}$ is normally distributed also seems reasonable.

Secondly, the raw data, $\left\{X_{i}\right\}$ and $\left\{Y_{i}\right\}$ are not equally reliable as functions of $i$. The $X_{i}$ 's and $Y_{i}$ 's become more reliable as $i$ increases since the adaptive process computes a better solution each time a new mesh is constructed. Therefore, the estimates for $c^{*}$ and $\theta$ should improve by placing more weight on the data as $i$ 


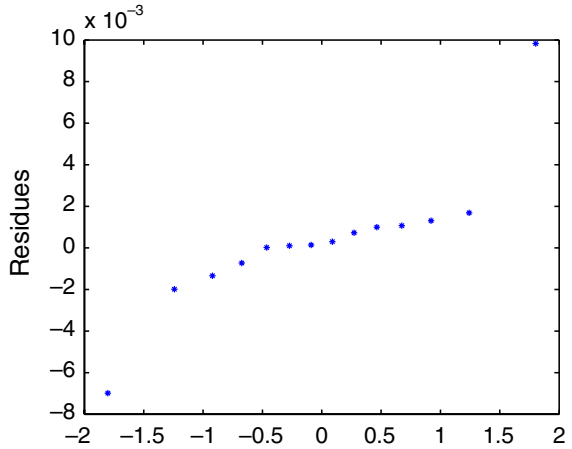

(a) Shapiro-Wilk W $0.7971 \mathrm{P}(\mathrm{W}) 0.004614$ cases

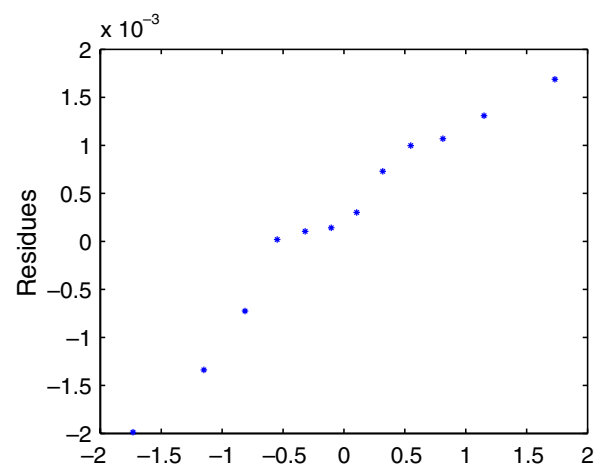

(b) Shapiro-Wilk W $0.9463 \mathrm{P}(\mathrm{W}) 0.584212$ cases

Fig. 2. Normal probability plot of residues. (a) Normal probability plot of residues and (b) normal probability plot of residues (two largest values omitted).

261 increases, provided the weights are properly chosen. So, the goal is to determine appropriate weights needed 262 to compensate for unequal data reliability so as to better estimate suitable values of $c^{*}$ and $\theta$ for Model A.

Model $\mathbf{A}$ is a transformation of a model of the form

$$
e \approx c \mathbf{R}_{1} \text {. }
$$

267 The variability of the residuals from the model (2.21) may be used as an indicator for the reliability of the 268 raw data. That is, the larger the variance associated with a data point the less reliable the data point, while 269 the smaller the variance the more reliable the data point. Based on this criteria we therefore construct 270 appropriate weights needed to account for unequal data reliability.

271 Following the approach in [8], let $x=c \mathbf{R}_{1}$, and define $e(x)$ as

$$
e(x):=x^{\theta}=c^{*} \mathbf{R}_{1}^{\theta},
$$

275 where $c^{*}=c^{\theta}$. A linear approximation of $e(x)$ about the point $x_{0}$ is given by

$$
e(x) \approx x_{0}^{\theta}+\theta x_{0}^{\theta-1}\left(x-x_{0}\right) .
$$

278 In the analysis of (2.22), assuming the residuals $r_{i}$ are normally distributed with mean zero and constant 279 variance $\sigma^{2}, N\left(0, \sigma^{2}\right)$, for the least squares parameter fit we minimize $\frac{1}{\sigma^{2}} \sum_{i=1}^{n} r_{i}^{2}$ (see (2.17)). However, in 280 (2.21) the $r_{i}$ 's are $N\left(0, \sigma_{i}^{2}\right), i=1, \ldots, n$ (i.e. not constant variance) hence for the least squares parameter 281 fit we minimize instead $\sum_{i=1}^{n} \frac{1}{\sigma^{2}} r_{i}^{2}$. Now, the least squares model corresponding to (2.22) may be written 282 as a weighted least squares model for (2.21) as

$$
\begin{aligned}
\frac{1}{\sigma^{2}} \sum_{i=1}^{n} r_{i}^{2} & =\sum_{i=1}^{n} \frac{1}{\sigma^{2}}\left(e_{i}(x)-x_{0_{i}}^{\theta}\right)^{2} \\
& \approx \sum_{i=1}^{n} \frac{1}{\sigma^{2}}\left(\theta x_{0_{i}}^{\theta-1}\left(x_{i}-x_{0_{i}}\right)\right)^{2} \\
& =\sum_{i=1}^{n} \frac{1}{\sigma^{2}} \theta^{2} x_{0_{i}}^{2(\theta-1)}\left(x_{i}-x_{0_{i}}\right)^{2} \\
& =\sum_{i=1}^{n} \frac{1}{\sigma^{2}} w_{i}\left(x_{i}-x_{0_{i}}\right)^{2} \\
& =\sum_{i=1}^{n} \frac{1}{\sigma_{i}^{2}}\left(x_{i}-x_{0_{i}}\right)^{2},
\end{aligned}
$$


285 where

$$
w_{i}=\frac{\theta^{2}}{x_{0_{i}}^{2(1-\theta)}}
$$

289 and

$$
\sigma_{i}^{2}=\frac{\sigma^{2}}{w_{i}}
$$

292 From (2.29) we have weights associated with the raw data reliability. Note that for equally reliable data

293 corresponding to $\theta=1, w_{i}=1$ for all $i$. On the other hand if $0<\theta<1$ or $\theta>1$, then clearly the $w_{i}$ 's are 294 not constant.

295 Thus, appropriate values for $c^{*}$ and $\theta$ for Model $\mathbf{A}$ with data reliability accounted for may be obtained 296 by minimizing

$$
\frac{1}{\sigma^{2}} \sum_{i=1}^{n} w_{i}\left(e_{i}-c^{*} \mathbf{R}_{1_{i}}^{\theta}\right)^{2}
$$

300 Since $x_{0}$ is an estimator for $e$,

$$
w_{i}=\frac{\theta^{2}}{e_{i}^{2(1-\theta)}}
$$

304 defines appropriate weights for minimizing (2.31).

305 As mentioned above, as the $e_{i}$ 's are unknown $c^{*}$ and $\theta$ must be obtained through Model $\mathbf{B}$.

306 Analogous to Model A, Model $\mathbf{B}$ is a transformation of a model of the form

$$
Y_{i} \approx c X_{i}:=\left|\mathbf{R}_{1_{i}}^{2}-\mathbf{R}_{1_{i+1}}^{2}\right|^{1 / 2} .
$$

309 From (2.11) and (2.12) we have

$$
Y_{i} \approx c^{*} \mathbf{R}_{1_{i}}^{\theta}\left(1-\frac{\eta\left(u_{i+1}, \Pi_{h, i+1}\right)^{2 \theta}}{\eta\left(u_{i}, \Pi_{h, i}\right)^{2 \theta}}\right)^{1 / 2} .
$$

312 Assuming that $\eta\left(u_{i+1}, \Pi_{h, i+1}\right) \approx \tilde{\mu} \eta\left(u_{i}, \Pi_{h, i}\right)$, for $0<\tilde{\mu}<1$ we have $Y_{i} \approx c^{*}\left(1-\tilde{\mu}^{2 \theta}\right)^{1 / 2} \mathbf{R}_{1_{i}}^{\theta}$, which is equiv313 alent to (2.22). Following the same approach used in arriving at (2.31) with Model B we minimize 314 equivalently

$$
\frac{1}{\sigma_{*}^{2}} \sum_{i=1}^{n} w_{*_{i}}\left(Y_{i}-c^{*} X_{i}(\theta)\right)^{2}
$$

318 where $\sigma_{*}^{2}$ is the variance associated with the Model $\mathbf{B}$ residuals, and $w_{*}$ the equivalent weights accounting 319 for unequal data reliability.

320 By analogy to (2.32),

$$
w_{*_{i}}=\frac{K}{Y_{i}^{2(1-\theta)}}
$$

324 defines appropriate weights for minimizing (2.35) while accounting for unequal data reliability. The con325 stant $K$ in (2.36) is chosen such that $\sum_{i} w_{*_{i}}=1$. 
Note that for the case $\theta>1$ the choice of weights (2.36) have the undesired property of assigning more weight to less reliable data. However if $\theta>1$, let $\vartheta=1 / \theta$ and in place of (2.22) consider instead

$$
e(y):=y^{\vartheta}=c \mathbf{R}_{1} .
$$

331 A linear approximation of the model transformation (2.37) then yields a similar choice for the weights,

$$
w_{*_{i}}=\frac{K}{Y_{i}^{2(1-\vartheta)}}
$$

satisfying the requirement of more weight to the more reliable data points.

In numerical simulations, the cases where $\theta \gg 1$ are rare. In most of the cases we have investigated, the value for $\theta$ fell within $0<\theta \leqslant 1$.

In summary, the corresponding weighted, relative least squares maximization problem for estimating optimal values for $c^{*}$ and $\theta$ is given by

$$
\max _{c^{*}>0, \theta>0}\left\{\mathscr{L}\left(c^{*}, \theta, w_{*}\right)\right\}=\max _{c^{*}>0, \theta>0}\left\{\left[\prod_{i=1}^{n}\left(\frac{w_{*_{i}}}{2 \pi}\right)^{1 / 2}\right] \exp \left[-\frac{1}{2} \sum_{i=1}^{n} w_{*_{i}}\left[\bar{Y}_{i}-c^{*} \bar{X}_{i}(\theta)\right]^{2}\right]\right\} .
$$

343 Note that the weights $w_{*}$ are aimed at accounting for unequal data reliability and not as normalizing factors 344 for unequal error variances.

345 The estimated values for $c^{*}$ and $\theta$ are then used in Model $\mathbf{A}$ to estimate the true error in each approx346 imate solution.

347 Below is a summary of the algorithm used:

348 Algorithm A

349 Given $u_{1}, u_{2}, \ldots, u_{n+1}$,

1. Using (2.6) and (2.13) generate $\left\{Y_{i}\right\}$ and $\left\{X_{i}(\theta)\right\}$ respectively.

2. Set $w_{i}=1$ and obtain parameter estimates for $c^{*}$ and $\theta$ from (2.39).

3. Using the current parameter estimate for $\theta$, construct a new set of weights based on $(2.36)$ or $(2.38)$.

4. Compute new parameter estimates from (2.39) using the new set of weights.

5. If the parameter estimates for $c^{*}$ and $\theta$ have converged, stop. Else, go to step 3.

In our investigations $c^{*}$ and $\theta$ in Algorithm $\mathrm{A}$ are usually found in less than 6 iterations through steps $3-$ 5.

\section{Numerical experiments}

360 In this section we present numerical results from applying Algorithm A to a posteriori error estimation. 361 For the first three examples we consider the Poisson equation where the solutions are chosen to test the 362 robustness of the method. For the second set of examples we consider a non-linear system of equations which arise in the modeling of viscoelastic fluid flow.

364 For comparison, for Examples 2 and 4 we include in Figs. 4 and 6(a) predicted errors (Predicted L.S.

365 Error) obtained from using the least squares fit for $Y_{i}=c^{*} X_{i}$, i.e. $c^{*}$ satisfying (2.17) for $\theta=1$. 


\subsection{The Poisson problem}

Consider the Poisson equation with homogeneous Dirichlet boundary conditions in a polygonal domain $\Omega \subset \mathbb{R}^{2}$ with boundary $\Gamma$

$$
-\Delta u=f \quad \text { in } \Omega, \text { and } \quad u=0 \text { on } \Gamma .
$$

372 Using the standard Galerkin approach, the continuous and discrete weak formulations of (3.40) are given 373 respectively as: find $u \in \mathscr{X}=H_{0}^{1}(\Omega):=\left\{v \in H^{1}(\Omega): v=0\right.$ on $\left.\Gamma\right\}$ such that

$$
\int_{\Omega} \nabla u \cdot \nabla v \mathrm{~d} A=\int_{\Omega} f v \mathrm{~d} A, \quad \forall v \in \mathscr{X}
$$

377

and find $u_{h} \in \mathscr{X}_{h} \subset \mathscr{X}=H_{0}^{1}(\Omega)$ such that

$$
\int_{\Omega} \nabla u_{h} \cdot \nabla v_{h} \mathrm{~d} A=\int_{\Omega} f v_{h} \mathrm{~d} A, \quad \forall v_{h} \in \mathscr{X}_{h} .
$$

381 In [2], Carstensen and Verfürth developed $H^{1}$-norm and $L^{2}$-norm a posteriori error estimates for problem 382 (3.40) for $u_{h}$ the linear finite element approximation, given by (3.42). They showed that the a posteriori er383 ror estimates are dominated by edge jump terms. By omitting the element residual in the standard residual 384 error estimator they proved the following theorem.

Theorem 3.1 [2]. Let $u$ and $u_{h}$ be the unique solutions to problems (3.41) and (3.42) respectively. There are constants $c_{1}$ and $c_{2}$ that depend on the shape regularity constant and on triangulation properties (see [2], Section 2) such that

$$
\left\|u-u_{h}\right\|_{1,2} \leqslant c_{1}\left\{\sum_{E} \eta_{E}^{2}\right\}^{1 / 2}+c_{2} \inf _{f_{h} \in \mathscr{X}_{h}}\left\{\sum_{T \in \Pi_{h, i}}|T|\left\|f-f_{h}\right\|_{0, T}^{2}\right\}^{1 / 2},
$$

391 where $\eta_{E}=h_{E}^{1 / 2}\left\|\left[\nabla u_{h} \cdot \mathbf{n}\right]_{E}\right\|_{0 ; E}$, and $|T|$ is the area of the triangle $T$.

The second term in (3.43) is the oscillation error term and in general is a higher order term and can be ignored in an adaptive procedure. Thus the error estimator above, with $c_{2}=0$, is of the form (2.3). Therefore, the true error can be estimated through a relationship of the form (2.5). We apply Algorithm A to estimate parameter values for $c^{*}$ and $\theta$, and then use these values to estimate the true error in a sequence of approximate solutions.

\subsubsection{Numerical examples}

Here we present three numerical examples based on the a posteriori error estimate in Theorem 3.1.

Example 1. In this example we let $u(x, y)=\left(1-\mathrm{e}^{\lambda x} \cos (2 \pi y)\right)^{2}+\left(\frac{\lambda}{2 \pi} \mathrm{e}^{\lambda x} \sin (2 \pi y)\right)^{2}$, with $\Omega$ defined as $\Omega:=(0,1) \times(-0.5,0.5)$. The right hand side, $f$, and the boundary condition are then determined from $u$. The parameter $\lambda$ is defined as $\lambda=\frac{R e^{2}}{2}-\left(\frac{R e^{2}}{4}+4 \pi^{2}\right)^{1 / 2}$ with $\operatorname{Re}=40$. Note that $u(x, y) \in C^{\infty}(\Omega)$.

Example 2. In this example we let $u(x, y)=\left(x^{2}+y^{2}\right)^{1 / 4} \sin \left[\frac{1}{2} \tan ^{-1}\left(\frac{x-y}{-x-y}\right)\right], f=0$, with an L-shaped domain defined as $\Omega=(-1,1) \times(-1,1)-(0,1) \times(0,1)$. The boundary data is then determined by restricting $u$ to the boundary $\partial \Omega$. Note that $\nabla u(x, y)$ has a square root singularity at the origin.

5 Example 3. In this example we let $u(x, y)=\tan ^{-1}\left[60\left(x^{2}+y^{2}-1.0\right)\right]$, with the domain $\Omega$ defined as $6:=(-1.25,1.25) \times(-1.25,1.25)$. The right hand side, $f$, and the boundary data are then determined from $u$. The solution $u(x, y)$ has a rapid transition across the curve $x^{2}+y^{2}=1$. 
Let Itr represent the $i$ th iterate in the sequence of approximate solutions, while $N$ represents the number 409 of degrees of freedom associated with the approximating linear system. For $u_{i}$ an approximation of $u$, we 410 denote the error in the $H^{1}$ norm as $E_{H^{1}}$, and the corresponding predicted error as $\widetilde{E}_{H^{1}}$. We also compute the 411 effectivity index, $I_{\text {eff }}$, as

$$
I_{\mathrm{eff}}:=\frac{\widetilde{E}_{H^{1}}}{E_{H^{1}}}
$$

414 Example 1 illustrates the method for the case of a smooth solution. The results presented in Table 1 and 415 Fig. 3 show that the true error is determined to within $2 \%$. For this example the approximations were gen416 erated using uniform refinements of the preceeding mesh. Similar results were obtained for approximations 417 from adaptively refined meshes.

418 The results for Example 2 are presented in Table 2 and Fig. 4. To illustrate the robustness of the method 419 the sequence of approximate solutions was generated using adaptively refined meshes. From the upper half 420 of Table 2 observe that the effectivity index oscillates between $75 \%$ and $98 \%$. The solution for this example 421 has a point singularity in the derivative of the true solution and demonstrates the need for Assumption II. 422 The mesh refinement algorithm (described in [6]) used in this study does not satisfy completely the condi423 tions of Assumption II. The refinement algorithm actually requires three levels of refinement to fully satisfy 424 the conditions of Assumption II. The point singularity in the derivative makes the solution highly sensitive

Table 1

Example 1: True error, predicted error, and effectivity index using uniform refinements $\left(c^{*}=0.2104, \theta=1.0216\right)$

\begin{tabular}{|c|c|c|c|}
\hline$N$ & $E_{H^{1}}$ & $\widetilde{E}_{H^{1}}$ & $I_{\mathrm{eff}}$ \\
\hline 676 & 0.51161 & 0.49696 & 0.97 \\
\hline 1301 & 0.38917 & 0.36819 & 0.95 \\
\hline 2601 & 0.25596 & 0.25419 & 0.99 \\
\hline 5101 & 0.19523 & 0.19108 & 0.98 \\
\hline 10,201 & 0.12785 & 0.12763 & 1.00 \\
\hline 20,201 & 0.09769 & 0.09660 & 0.99 \\
\hline 40,401 & 0.06387 & 0.06349 & 0.99 \\
\hline 80,401 & 0.04886 & 0.04820 & 0.99 \\
\hline 160,801 & 0.03192 & 0.03143 & 0.98 \\
\hline 320,801 & 0.02443 & 0.02390 & 0.98 \\
\hline
\end{tabular}

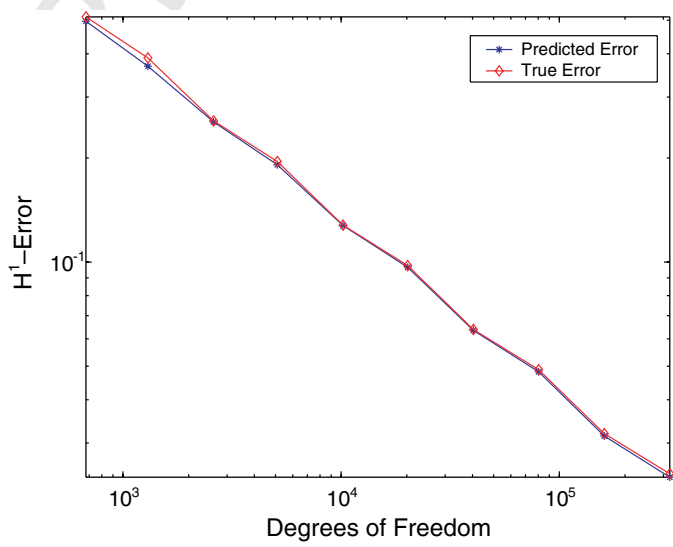

Fig. 3. Example 1: True error and predicted error vs. degrees of freedom using uniform refinements. 
Table 2

Example 2: True error, predicted error, and effectivity index using adaptive refinements $\left(c^{*}=0.2177, \theta=0.9315\right)$

\begin{tabular}{rrlll}
\hline Itr & \multicolumn{1}{l}{ N } & $E_{H^{1}}$ & $\widetilde{E}_{H^{1}}$ & $I_{\text {eff }}$ \\
\hline 1 & 645 & 0.16138 & 0.15794 & 0.98 \\
2 & 771 & 0.13514 & 0.10171 & 0.75 \\
3 & 932 & 0.11600 & 0.11310 & 0.97 \\
4 & 1149 & 0.09715 & 0.07420 & 0.76 \\
13 & 7567 & 0.02398 & 0.02372 & 0.99 \\
14 & 9301 & 0.02051 & 0.01781 & 0.87 \\
15 & 11,428 & 0.01791 & 0.01786 & 1.00 \\
16 & 13,989 & 0.01543 & 0.01384 & 0.90 \\
26 & 98,163 & 0.00440 & 0.00443 & 1.01 \\
27 & 118,651 & 0.00395 & 0.00412 & 1.04 \\
28 & 143,395 & 0.00353 & 0.00360 & 1.02 \\
29 & 173,195 & 0.00318 & 0.00332 & 1.04 \\
30 & 209,173 & 0.00287 & 0.00294 & 1.03 \\
\end{tabular}

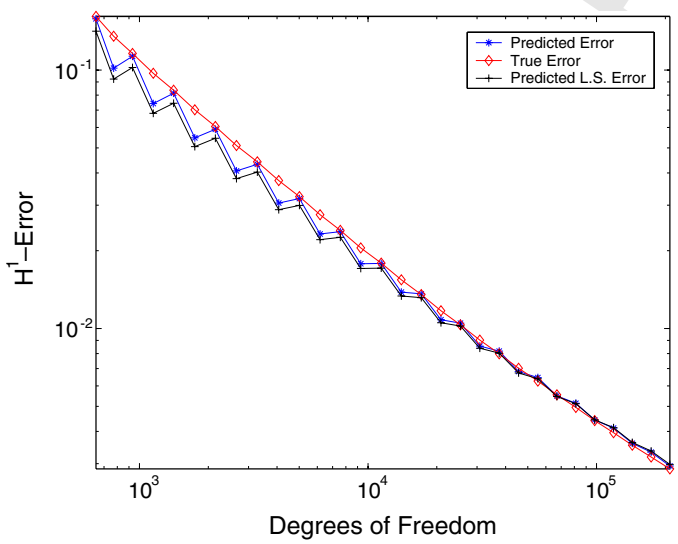

Fig. 4. Example 2: True error, predicted error and predicted 1.s. error $\left(c^{*}=0.2218\right)$ vs. degrees of freedom using adaptive refinements.

425 to the orientation of mesh elements. A single level of refinement may therefore add very little to the approx426 imating finite element space, while at the same time resulting in an increase in the value of $\mathbf{R}_{1}$. As shown in 427 Fig. 4, this phenomenon may lead to oscillations in the predicted errors. As in the convection-dominated 428 problems a flow-oriented mesh can remedy this effect (see [5]). Alternatively, we can just proceed with the 429 solution process until these mesh effects are suitably diminished as $h \rightarrow 0$. With the mesh effects diminished, 430 we may improve the predicted errors by truncating the data eliminating the highly oscillatory part of the 431 data. For this example, $c^{*}$ and $\theta$ are predicted using the last 15 data values. These parameter values are 432 then used in predicting the errors in Table 2 and Fig. 4.

433 Example 3 investigates another important phenomenon; non-physical oscillations in the approximate 434 solutions. Starting with a coarse mesh, and a strongly varying forcing term, non-physical oscillations occur 435 in the approximate solutions. These oscillations make the error estimators highly unreliable at the begin436 ning of the adaptive process, since $\mathbf{R}_{3}$ and $\mathbf{R}_{4}$ are of the same order as $\mathbf{R}_{1}$ (and $\mathbf{R}_{2}$ ). As in Example 2, accu437 rate error predictions require that the adaptive procedure sufficiently refine the mesh to make the current 438 error estimator $\left(\mathbf{R}_{1}\right)$ much larger than data oscillation $\left(\mathbf{R}_{3}+\mathbf{R}_{4}\right)$, thus satisfying the initial assumption that 439 $\mathbf{R}_{3}$ and $\mathbf{R}_{4}$ are higher order terms relative to $\mathbf{R}_{1}\left(\right.$ and $\mathbf{R}_{2}$ ). So, for Example 3 the data used in estimating the 
440 parameters $c^{*}$ and $\theta$, corresponds to the data from iterations 12-20 of the adaptive process. These param441 eter values are then used in predicting the errors in Table 3 and Fig. 5(b). Fig. 5(a) shows the predicted 442 errors when all the data is used in estimating the parameter values $c^{*}$ and $\theta$.

\section{3.2. The Oldroyd-B model for viscoelastic fluid flow}

444 In this section we apply Algorithm A to a viscoelastic fluid flow problem. Consider an incompressible, 445 time independent, creeping, isothermal viscoelastic fluid flowing in a bounded, connected, open domain $446 \Omega \subset \mathbb{R}^{n}(n=2,3)$, with Lipschitz boundary $\Gamma=\partial \Omega$ and $\Gamma_{\text {in }} \subset \Gamma$ denoting the inflow boundary. The system 447 of governing equations for such a fluid flow satisfying an Oldroyd-B constitutive law is given by

Table 3

Example 3: Using the bottom 9 data points we estimate a value for $c^{*}\left(c^{*}=0.2244\right)$ and $\theta(\theta=0.9864)$

\begin{tabular}{rrrrr}
\hline Itr & \multicolumn{1}{l}{$N$} & $E_{H^{1}}$ & $\widetilde{E}_{H^{1}}$ & $I_{\text {eff }}$ \\
\hline 1 & 441 & 63.1496 & 22.5052 & 0.36 \\
2 & 597 & 52.3591 & 16.2876 & 0.31 \\
5 & 1609 & 20.1439 & 10.9946 & 0.55 \\
6 & 2259 & 15.5921 & 10.3009 & 0.66 \\
9 & 6457 & 6.93781 & 6.50190 & 0.94 \\
10 & 9525 & 5.47825 & 5.28891 & 0.97 \\
13 & 35,032 & 2.71624 & 2.71389 & 1.00 \\
14 & 55,764 & 2.16260 & 2.16196 & 1.00 \\
17 & 203,958 & 1.16419 & 1.16166 & 1.00 \\
18 & 302,110 & 0.95926 & 0.95238 & 0.99 \\
19 & 459,369 & 0.77522 & 0.77675 & 1.00 \\
20 & 682,542 & 0.64805 & 0.64386 & 0.99 \\
\end{tabular}

These values are then used in predicting the true error.
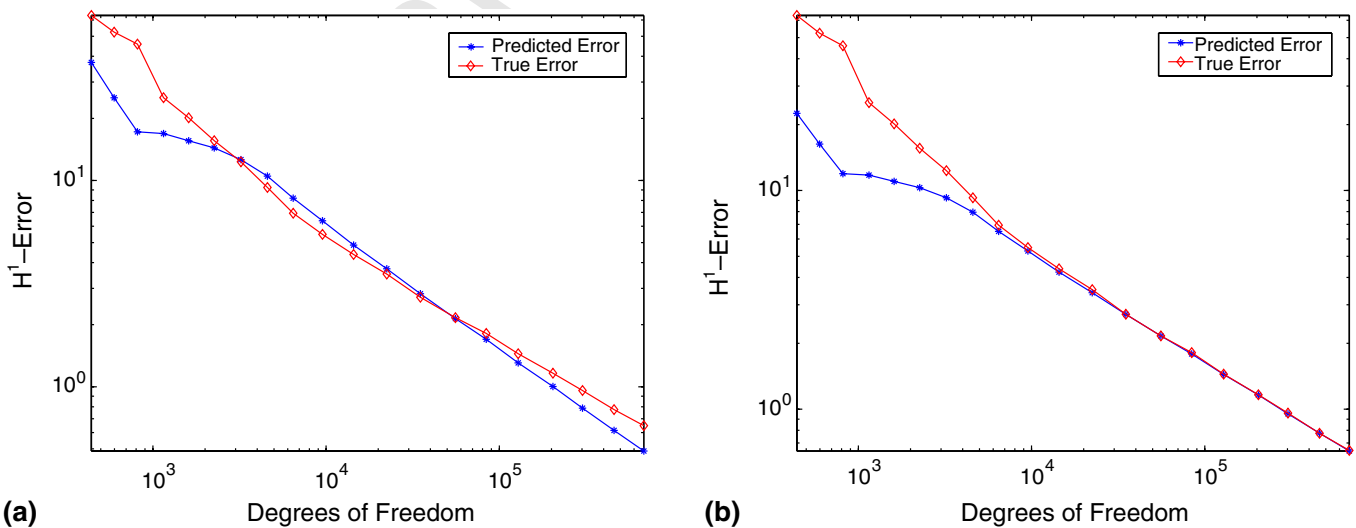

Fig. 5. Example 3: True error and predicted error vs. degrees of freedom. (a) Using all the data to estimate $c^{*}$ and $\theta$ and (b) truncated data used in estimating $c^{*}$ and $\theta$. 

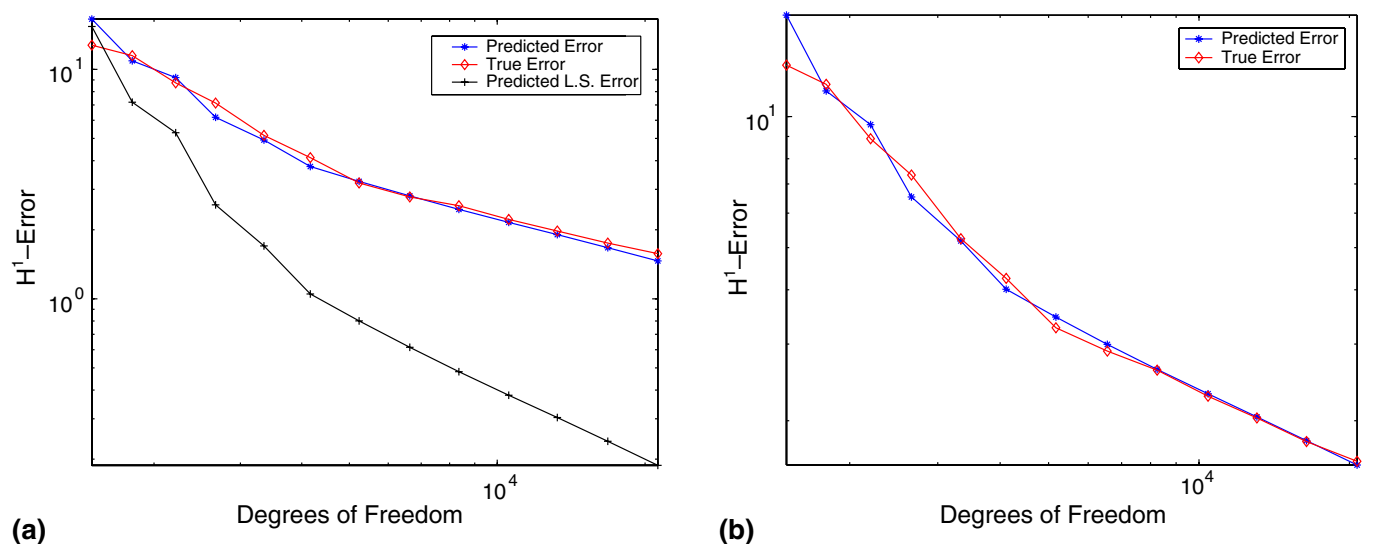

Fig. 6. Example 4: Plots of the true error, predicted error and predicted 1.s. error vs. degrees of freedom. (a) $\lambda=0.1$ : True error, predicted error $\left(c^{*}=7.6719, \theta=0.5512\right)$, and predicted 1.s. error $\left(c^{*}=3.8039\right)$ and $(\mathrm{b}) \lambda=1.0$ : True error and predicted error $\left(c^{*}=7.4809, \theta=0.5436\right)$.

$$
\begin{aligned}
& \tau+\lambda\left[(u \cdot \nabla) \tau-(\nabla u)^{\mathrm{T}} \tau-\tau \nabla u\right]-2 \alpha \mathbf{D}(u)=0 \quad \text { in } \Omega, \\
& -2(1-\alpha) \nabla \cdot \mathbf{D}(u)-\nabla \cdot \tau+\nabla p=f \quad \text { in } \Omega, \\
& \nabla \cdot u=0 \quad \text { in } \Omega, \\
& u=u_{0} \quad \text { on } \Gamma, \\
& \tau=\tau_{0} \quad \text { on } \Gamma_{\text {in }},
\end{aligned}
$$

451 where $\tau$ is the viscometric stress tensor, $u$ is the fluid velocity, $p$ is the pressure, $\lambda$ is the Weissenberg ${ }^{1}$ number, $\mathbf{D}(u)=\frac{1}{2}\left(\nabla u+(\nabla u)^{\mathrm{T}}\right)$ is the deformation tensor, and $\alpha$ is a model parameter.

453 Remark. In the case $u_{0}=0, \Gamma_{\text {in }}=\emptyset$, hence no boundary condition for the stress is necessary.

Let $\left[u_{h}, \tau_{h}, p_{h}\right] \in \mathscr{X}_{h} \subset \mathscr{X}:=\left[\left(H_{0}^{1}(\Omega)\right)^{n} \times\left(H^{1}(\Omega)\right)_{\mathrm{sym}}^{n \times n} \times L_{0}^{2}(\Omega)\right]$, where $\left(H^{1}(\Omega)\right)_{\mathrm{sym}}^{n \times n}$ is a symmetric tensor space of square integrable functions whose gradients are also square integrable, $L_{0}^{2}(\Omega)$ is the scalar space of square integrable functions with mean zero. For our computations we use $\mathscr{X}_{h}:=\left[\left(\mathbb{P}_{2}\right)^{n} \times\right.$ $\left.\left(\mathbb{P}_{1}\right)_{\text {sym }}^{n \times n} \times \mathbb{P}_{1}\right]$ where $\mathbb{P}_{k}$ is the space of continuous piecewise polynomials of order $k$.

459 tive equation (3.44), we have a variational form for the system (3.44)-(3.47) (with $u_{0}=0$ ) given by

$$
\begin{aligned}
\left\langle F_{h}\left(\left[u_{h}, \tau_{h}, p_{h}\right]\right),\left[v_{h}, \sigma_{h}, q_{h}\right]\right\rangle: & =\int_{\Omega}\left(\tau_{h}+\lambda\left[\left(u_{h} \cdot \nabla\right) \tau_{h}-\left(\nabla u_{h}\right)^{\mathrm{T}} \tau_{h}-\tau_{h} \nabla u_{h}\right]-2 \alpha \mathbf{D}\left(u_{h}\right)\right):\left(\sigma_{h}+\delta\left(h_{T}, u_{h}\right) u_{h} \cdot \nabla \sigma_{h}\right) \mathrm{d} A \\
& +\int_{\Omega}\left(\left(2(1-\alpha) \mathbf{D}\left(u_{h}\right)+\tau_{h}-p_{h} \rrbracket\right): \nabla v_{h}-f \cdot v_{h}\right) \mathrm{d} A \\
& +\int_{\Omega} q_{h} \nabla \cdot u_{h} \mathrm{~d} A, \quad \forall\left[v_{h}, \sigma_{h}, q_{h}\right] \in X_{h},
\end{aligned}
$$

462 where

$$
\delta\left(h_{T}, u_{h}\right)= \begin{cases}\frac{c h_{T}|T|^{1 / 2}}{\lambda\left\|u_{h}\right\|_{0 ; T}}, & \text { if } u_{h} \neq 0 \text { on } T \\ 0, & \text { if } u_{h}=0 \text { on } T\end{cases}
$$

\footnotetext{
${ }^{1}$ The Weissenberg number represents a measure of the ratio of the magnitude of the elastic forces to that of the viscous forces [1].
} 
where $|T|$ represents the area/volume of $T$.

Using the general framework in [9], a residual based a posteriori error estimate has been constructed in [4] for the system (3.44)-(3.47) (with $u_{0}=0$ ) as summarized in the following theorem.

Theorem 3.2 [4]. Assume that there exists subspaces $X_{D} \subset X, Y_{D}^{*} \subset Y^{*}$ such that the variational solution, $[u, \tau, p]$, of (3.44)-(3.47) (with $u_{0}=0$ ) satisfies $[u, \tau, p] \in X_{D}$ and $D F([u, \tau, p]) \in \operatorname{Isom}\left(X_{D}, Y_{D}^{*}\right)$. Then, for $\left.u_{h}, \tau_{h}, p_{h}\right] \in X_{h} \subset X_{D}$, sufficiently close to $[u, \tau, p]$, we have the following a posteriori error estimate.

$$
\begin{aligned}
\left\{\left\|u-u_{h}\right\|_{1,2}^{2}+\left\|\tau-\tau_{h}\right\|_{1,2}^{2}+\left\|p-p_{h}\right\|_{0,2}^{2}\right\}^{1 / 2} \leqslant & c_{1}\left\{\sum_{T} \eta_{T}^{2}\right\}^{1 / 2}+c_{2}\left\{\sum_{T}\left(\left\|u_{h}\right\|_{\infty, T} \delta\left(h_{T}, u_{h}\right)\right)^{2}\left\|R_{s}\right\|_{0,2 ; T}^{2}\right\}^{1 / 2} \\
& +c_{3}\left\{\sum_{T} h_{T}^{2}\left\|f-\pi_{k, T} f\right\|_{0,2 ; T}^{2}\right\}^{1 / 2}
\end{aligned}
$$

474 where

$$
\begin{aligned}
R_{s}:= & \tau_{h}+\lambda\left((u \cdot \nabla) \tau-(\nabla u)^{\mathrm{T}} \tau-\tau \nabla u\right)-2 \alpha \mathbf{D}\left(u_{h}\right), \\
\eta_{T}:= & \left\{h_{T}^{2}\left\|\tau_{h}+\lambda\left((u \cdot \nabla) \tau-(\nabla u)^{\mathrm{T}} \tau-\tau \nabla u\right)-2 \alpha \mathbf{D}\left(u_{h}\right)\right\|_{0,2 ; T}^{2}\right. \\
& +h_{T}^{2}\left\|-\nabla \cdot \tau_{h}-2(1-\alpha) \nabla \cdot \mathbf{D}\left(u_{h}\right)+\nabla p_{h}-\pi_{k, T} f\right\|_{0,2 ; T}^{2}+\left\|\nabla \cdot u_{h}\right\|_{0,2 ; T}^{2} \\
& \left.+h_{E}\left\|\left[\tau_{h} \cdot \mathbf{n}_{E}-p_{h} \mathbf{n}_{E}+2(1-\alpha) \mathbf{D}\left(u_{h}\right) \cdot \mathbf{n}_{E}\right]_{E}\right\|_{2 ; E}^{2}\right\}^{1 / 2}
\end{aligned}
$$

480 and $\pi_{k, T} f$ is a projection of $f$ onto a polynomial space with degree $k$ on the mesh element $T$.

Note that for $\lambda=0$, the system (3.44)-(3.47) reduces to a Stokes problem commonly referred to as the Stokes-Oldroyd problem. Here we only consider the case $\lambda>0$.

Observe that the second term on the right hand side of (3.50) can be bounded by the first term. Also, as commented in Section 2, the third term is a higher order term. Thus we use for our a posteriori error estimate (3.50) with $c_{1}=c^{*}, c_{2}=c_{3}=0$, which fits the framework discussed in Section 2.

\subsubsection{Numerical examples}

In this section we present numerical results based on the a posteriori error estimate (3.50) with $c_{1}=c^{*}$ and $c_{2}=c_{3}=0$. Let $E$ represent the total error associated with the approximations to velocity, pressure and stress defined as

$$
E:=\left\{\left\|u-u_{h}\right\|_{H^{1}}^{2}+\left\|p-p_{h}\right\|_{L^{2}}^{2}+\left\|\tau-\tau_{h}\right\|_{H^{1}}^{2}\right\}^{1 / 2},
$$

492 while the corresponding predicted error is denoted by $\widetilde{E}$. The effectivity index is then computed as $493 I_{\mathrm{eff}}=\widetilde{E} / E$.

494 Example 4. For this example $\Omega$ is an L-shaped domain given by $\Omega=(-1,1) \times(-1,1)-(0,1) \times(0,1)$. The 495 velocity, polymeric stress, and pressure used are

$$
u(x, y):=\left[\frac{(y-0.1)}{\frac{\left[(x-0.1)^{2}+(y-0.1)^{2}\right]^{1 / 2}}{\left[(x-0.1)^{2}+(y-0.1)^{2}\right]^{1 / 2}}}\right], \quad \tau:=2 \alpha \mathbf{D}(u), \quad p(x, y):=(2-x-y)^{1 / 2} .
$$

498 Note that the solution has a point singularity at $(0.1,0.1)$ which lies just outside the domain (near an incom499 ing corner). 
Table 4

Example 4: True error, predicted error, and effectivity index

\begin{tabular}{|c|c|c|c|c|c|c|}
\hline \multirow[t]{2}{*}{ Itr } & \multicolumn{3}{|c|}{$\begin{array}{l}\lambda=0.1 \\
c^{*}=7.6719, \theta=0.5512\end{array}$} & \multicolumn{3}{|c|}{$\begin{array}{l}\lambda=1.0 \\
c^{*}=7.4809, \theta=0.5436\end{array}$} \\
\hline & $E$ & $\widetilde{E}$ & $I_{\mathrm{eff}}$ & $E$ & $\widetilde{E}$ & $I_{\text {eff }}$ \\
\hline 1 & 12.7522 & 16.5730 & 1.30 & 13.1271 & 17.1165 & 1.30 \\
\hline 2 & 11.4822 & 10.9016 & 0.95 & 11.8647 & 11.4589 & 0.97 \\
\hline 3 & 8.73590 & 9.20858 & 1.05 & 8.89702 & 9.58332 & 1.08 \\
\hline 4 & 7.13155 & 6.17872 & 0.87 & 7.34208 & 6.53529 & 0.89 \\
\hline 5 & 5.15579 & 4.92267 & 0.95 & 5.24249 & 5.17914 & 0.99 \\
\hline 6 & 4.12506 & 3.77315 & 0.91 & 4.24808 & 4.00993 & 0.94 \\
\hline 7 & 3.19656 & 3.24980 & 1.02 & 3.26976 & 3.46253 & 1.06 \\
\hline 8 & 2.78102 & 2.80875 & 1.01 & 2.89121 & 2.99519 & 1.04 \\
\hline 9 & 2.54572 & 2.45363 & 0.96 & 2.61384 & 2.62395 & 1.00 \\
\hline 10 & 2.21868 & 2.15377 & 0.97 & 2.27687 & 2.30663 & 1.01 \\
\hline 11 & 1.97436 & 1.90444 & 0.96 & 2.02980 & 2.04091 & 1.01 \\
\hline 12 & 1.74899 & 1.66938 & 0.95 & 1.79076 & 1.80031 & 1.01 \\
\hline 13 & 1.57410 & 1.46191 & 0.93 & 1.61318 & 1.58253 & 0.98 \\
\hline
\end{tabular}

Using $\eta_{T}$, given by (3.52), as an error indicator for the error on each element $T$ of the mesh $\Pi_{h, i}$, a 501 sequence of adaptively generated approximations was generated for $\lambda=0.1$ and $\lambda=1.0$. Using Algorithm A 502 corresponding values for $c^{*}$ and $\theta$ were then computed. Estimates for the error in the approximations were 503 then found. The results are summarized in Table 4 and Fig. 6. Note that in (3.44)-(3.48) as $\lambda$ increases the 504 system becomes more non-linear. For both values of $\lambda$ accurate estimates for the error were obtained.

505 Example 5. For this example we consider a benchmark problem in viscoelastic fluid flow simulation; chan506 nel flow with a cylindrical obstacle [3]. The ratio of the channel height to the cylinder diameter, $H$, is taken 507 to be 4 , while the maximum inflow velocity is set at 1.5 . The boundary conditions imposed are as follows. 508 For velocity: a fully developed flow field (parabolic profile) at the inflow and outflow boundaries, and a 509 non-slip $(u=0)$ condition along the other boundaries. For the polymetric stress: along the inflow boundary 510 the polymetric stress for a fully developed channel flow is assumed. For pressure: the pressure is fixed at one of the inflow mesh points to zero. There does not exist a closed form solution to this problem.

As described for Example 4, a sequence of approximate solutions was adaptively generated for $\lambda=0.1$ and $\lambda=0.5$, and corresponding values for $c^{*}$ and $\theta$ computed Table 5.

514 Presented in Fig. 7 are graphs of the estimated error and the a posteriori error estimator $\left(c_{1}=1\right.$, $515 c_{2}=c_{3}=0$ ) versus the degrees of freedom. The graphs are similar to those for Example 4 for which we 516 know the true solution.

\section{3.3. Comment on the numerical experiments}

518 As previously commented, the asymptotic value for $\theta$ in the model (2.5) is 1 . For Examples 1-3, which 519 are linear, the non-linear, weighted least-squares algorithm computed values of $1.02,0.93$, and 0.99 , respectively, for $\hat{\theta}$. For the non-linear Examples 4 and 5, the values computed for $\hat{\theta}$ were 0.59 and 0.60 , respectively, indicating that the approximations are still quite far away from following their asymptotic behavior. Nonetheless, as demonstrated by Example 4 for which the true solution is known, the described procedure was able to accurately predict the error. 
Table 5

Channel flow problem. Example 5: Predicted errors for $\lambda=0.1$ and $\lambda=0.5$

\begin{tabular}{rlr}
\hline Itr & $\lambda=0.1$ & \multicolumn{2}{c}{$\lambda=0.5$} \\
& $\widetilde{E}_{H^{1}}$ & 18.7954 \\
\hline 1 & 6.30229 & 14.3301 \\
2 & 4.87879 & 12.0718 \\
3 & 4.35150 & 9.13656 \\
4 & 3.08777 & 7.68084 \\
5 & 2.59562 & 6.38090 \\
6 & 2.21672 & 5.63922 \\
7 & 1.90468 & 4.90932 \\
8 & 1.67350 & 4.32252 \\
9 & 1.46767 & 3.73712 \\
10 & 1.26813 & 3.24057 \\
11 & 1.09925 & 2.85818 \\
12 & 0.98498 & 2.55865 \\
13 & 0.89483 & \\
\hline
\end{tabular}

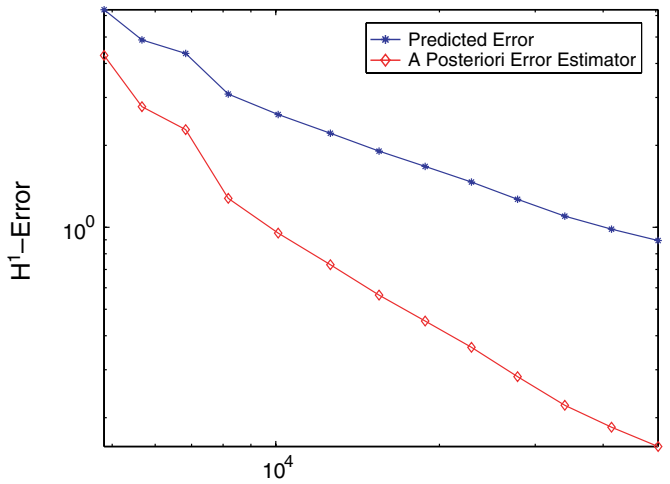

(a)

Degrees of Freedom

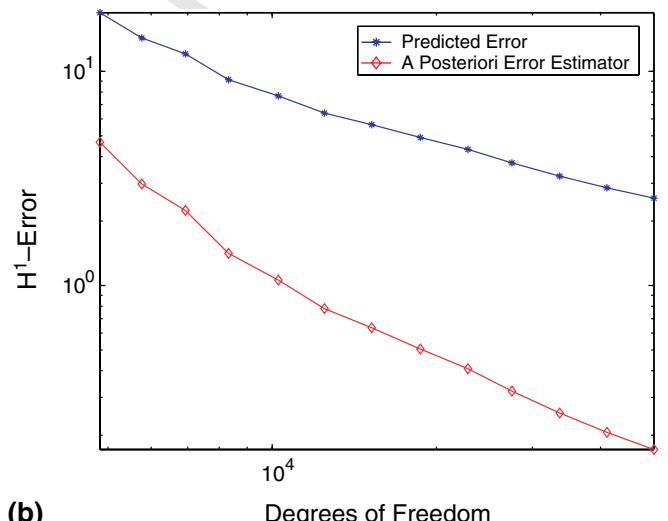

Fig. 7. Example 5: Predicted error and a posteriori error estimator $\left(c_{1}=1, c_{2}=c_{3}=0\right)$ vs. degrees of freedom. (a) $\lambda=0.1$ : Predicted error $\left(c^{*}=2.6716, \theta=0.5902\right)$, and the a posteriori error estimator and (b) $\lambda=0.5$ : Predicted error $\left(c^{*}=7.4177, \theta=0.6036\right)$, and the a posteriori error estimator.

\section{Acknowledgment}

525 This work was partially supported by the ERC Program of the National Science Foundation under 526 Award Number ERC-9731680.

\section{References}

528 [1] R.B. Bird, R.C. Armstrong, O. Hassager, Dynamics of Polymeric Liquids, John Wiley and Sons, Inc., 1987.

529 [2] C. Carstensen, R. Verfürth, Edge residuals dominate a posteriori error estimates for low order finite element methods, SIAM J. $530 \quad$ Numer. Anal. 36 (1999) 1571-1587.

531 [3] B. Caswell, Report on the IX international workshop on numerical methods in non-Newtonian flows, J. Non-Newton. Fluid Mech. 532 62 (1996) 99-110. 
533 [4] V.J. Ervin, L.N. Ntasin, A posteriori error estimation and adaptive computation of viscoelastic fluid flow. Numer. Methods Partial 534 Differen. Equat. Available from <http://www.math.clemson.edu/ vjervin/papers/erv023.pdf>, 2002.

535 [5] T. Iliescu, A flow-aligning algorithm for convection-dominated problems, Int. J. Numer. Meth. Engng. 46 (1999) $993-1000$.

536 [6] J. Maubach, Local bisection refinement for simplical grids generated by reflection, SIAM J. Sci. Comput. 16 (1995) $210-227$.

537 [7] P. Morin, R.H. Nochetto, K.G. Siebert, Convergence of adaptive finite element methods, SIAM Rev. 44 (4) (2002) 631-658.

538 [8] G.A.F. Seber, C.J. Wild, Nonlinear Regression, Wiley, 1989.

539 [9] R. Verfürth, A Review of a Posteriori Error Estimation and Adaptive Mesh Refinement Techniques, Wiley, Teubner, 1996. 